\title{
Expression of the ID01/TD02-AhR pathway in tumor cells or the tumor microenvironment is associated with Merkel cell polyomavirus status and prognosis in Merkel cell carcinoma
}

\author{
Lusi Oka Wardhani MD ${ }^{a, b, *}$, Michiko Matsushita CT, PhD ${ }^{a, c}$, Takeshi Iwasaki MD ${ }^{d}$, \\ Satoshi Kuwamoto MD, $\mathrm{PhD}^{\mathrm{a}}$, Daisuke Nonaka MD, PhD ${ }^{\mathrm{e}, \mathrm{f}}{ }^{\prime}$, Keiko Nagata MD, PhD ${ }^{\mathrm{a}}$, \\ Masako Kato MD, PhD ${ }^{a}$, Yukisato Kitamura MD, PhD ${ }^{c}$, Kazuhiko Hayashi MD, PhD ${ }^{a, *}$
}

\author{
a Division of Molecular Pathology, Department of Pathology, Tottori University Faculty of Medicine, Yonago 683-8503, Japan \\ ${ }^{\mathrm{b}}$ Clinical Pathology Department, Medical Faculty of Sebelas Maret University, Surakarta 57126, Indonesia \\ ${ }^{\mathrm{c}}$ Department of Pathobiological Science and Technology, School of Health Science, Tottori University Faculty of Medicine, \\ Yonago 683-8503, Japan \\ ${ }^{\mathrm{d} D e p a r t m e n t ~ o f ~ A n a t o m i c ~ P a t h o l o g y, ~ G r a d u a t e ~ S c h o o l ~ o f ~ M e d i c a l ~ S c i e n c e s, ~ K y u s h u ~ U n i v e r s i t y, ~ H i g a s h i-k u, ~ F u k u o k a ~ 812-8582, ~}$ \\ Japan \\ 'Department of Cellular Pathology, The Guy's and St Thomas' NHS Foundation Trust, London, SE11, United Kingdom \\ ${ }^{\mathrm{f}}$ The Christie Hospital and University of Manchester, Manchester M20 4BX, United Kingdom
}

Received 12 July 2018; revised 31 August 2018; accepted 6 September 2018

Keywords:

Merkel cell carcinoma; Merkel cell polyomavirus; Indoleamine 2,3-dioxygenase 1;

Tryptophan 2,3-dioxygenase 2 ;

Aryl hydrocarbon receptor
Summary Merkel cell carcinoma (MCC) is a rare, aggressive neuroendocrine skin cancer, with approximately $80 \%$ of cases related to Merkel cell polyomavirus (MCPyV). Indoleamine 2,3-dioxygenase 1 (IDO1) and tryptophan 2,3-dioxygenase 2 (TDO2) are the key rate-limiting enzymes of the tryptophanto-kynurenine metabolic pathway. With aryl hydrocarbon receptor (AhR), an intracellular transcription factor, they play a role in escaping the immunosurveillance process in several cancers. IDO1/TDO2/AhR expression associated with the MCPyV status and prognosis in MCC was investigated. Samples included 24 MCPyV-positive MCCs, 12 MCPyV-negative MCCs with squamous cell carcinoma, and $7 \mathrm{MCPyV}$ negative pure MCCs. They were stained immunohistochemically with IDO1, TDO2, and AhR antibodies and analyzed. Higher IDO1 expression in MCC tumor cells was found in MCPyV-negative than in MCPyV-positive MCC $(P<.001)$. The tumor microenvironment (TME) in MCPyV-negative MCC expressed higher TDO2 than in MCPyV-positive MCC $(P<.001)$. Kaplan-Meier and log-rank tests showed that MCC with lower IDO1 expression in tumor cells and with lower TDO2 and AhR expressions in TME had better overall survival than otherwise $(P=.043, .008$, and .035 , respectively); lower TDO2 expression in

\footnotetext{
is Competing interest: There is no conflict of interests.

论经 Funding/support: This study was supported, in part, by grants from Japan Society for the Promotion of Science (No. 26460433 [K.H.] and No. 26860238 [M.M.]).

* Corresponding authors at: Division of Molecular Pathology, Department of Pathology, Tottori University Faculty of Medicine, Nishi-cho 86, Yonago, Tottori 683-8503, Japan.

E-mail addresses: lusioka@gmail.com (L. O. Wardhani), michikohavefun@gmail.com (M. Matsushita), iwasakit-path@umin.ac.jp (T. Iwasaki), s.quamoto@gmail.com (S. Kuwamoto),dnonaka@msn.com (D. Nonaka), nanamon@theia.ocn.ne.jp (K. Nagata), makato@med.tottori-u.ac.jp (M. Kato), nshyk@med.tottori-u.ac.jp (Y. Kitamura), hayashik@med.tottori-u.ac.jp (K. Hayashi).
} 
TME was also associated with longer disease-specific survival $(P=.016)$. This suggests that IDO1, TDO2, and AhR express differentially in tumor cells or TME and play different roles in tumorigenesis between MCPyV-positive and MCPyV-negative MCC that may affect the MCC biology. Evaluating IDO1/TDO2/ $\mathrm{AhR}$ expression is important for selecting the most likely patients with MCC for immunotherapies targeting the IDO1/TDO2-AhR pathway.

(C) 2018 Elsevier Inc. All rights reserved.

\section{Introduction}

Merkel cell carcinoma (MCC) is an aggressive, neuroendocrine, cutaneous cancer generally diagnosed in elderly individuals with sun exposure, and its risk of occurrence is related to an immunocompromised condition and the presence of other cancers [1,2]. Approximately $80 \%$ of MCC cases are associated with Merkel cell polyomavirus (MCPyV). Integration of the monoclonal pattern of the viral genome into the tumor genome indicates that $\mathrm{MCPyV}$ infection and genomic integration occur earlier in tumorigenesis [3]. The presence of MCPyV infection is associated with histologic differences in MCCs. MCPyV-positive MCC tumor cells have uniform round nuclei and less cytoplasm, whereas MCPyV-negative cells have irregular nuclei and abundant cytoplasm [4]. MCPyV infection also contributes to a different survival prognosis. Patients with MCPyV-positive MCC generally showed longer survival and better prognosis than did those with MCPyV-negative MCC [5-8].

Recently, 3 phase II open-label clinical trials of therapeutic antibodies against programmed death 1 (PD1) or programmed death-ligand 1 (PD-L1), key targets of an immune-checkpoint pathway, were studied in patients with advanced-stage MCC. The trials demonstrated higher and more durable response rates than chemotherapy; however, a substantial number of advanced-stage MCC cases do not respond to PD1-PD-L1 inhibitors [9-11].

The tumor microenvironment (TME) is the cellular environment in which the tumor exists, including the surrounding blood vessels, fibroblasts, immune cells (lymphocytes, myeloid-derived suppressor cells, dendritic cells [DCs], and tumor-associated macrophages), signaling molecules, and the extracellular matrix [12]. The responders to cancer immunotherapy seem to carry an inflammatory T-cell signature in their TME: functional neoantigen presentation by DCs and infiltration and proliferation of tumor-specific cytotoxic T lymphocytes (CTLs). Therefore, novel strategies capable of transforming the immunosuppressive TME of non-T-cell inflammatory tumors to inflammatory tumors may support a new paradigm in cancer immunotherapy [12]. In the spotlight is the indoleamine 2,3-dioxygenase 1 (IDO1)/tryptophan 2,3dioxygenase 2 (TDO2)-kynurenine (KYN)-aryl hydrocarbon receptor (AhR) signaling pathway, which includes the tryptophan (Trp) catabolic enzymes IDO1 and TDO2, their product $\mathrm{KYN}$, and an endogenous ligand of the AhR. Recent research has revealed that KYN and AhR are key signaling molecules that can transduce the immunosuppressive effects of IDO1 and TDO2. In addition, IDO1 has been shown to participate in mechanisms of resistance to checkpoint inhibitors. Therefore, the combination of an IDO1 inhibitor with a checkpoint inhibitor is a promising strategy to expand patient populations for immunotherapy [12].

An essential amino acid in humans, Trp is not only necessary for protein synthesis but also used as a substrate in the metabolic synthesis of essential signaling molecules in physiology, the pathological system, and immunity [13]. Trp metabolism, which occurs mostly via the KYN pathway, produces several catabolic products with diverse biological activities. This process is catalyzed by IDO and TDO2, and its product, $N$-formyl KYN, is rapidly converted into KYN. IDO also catalyzes the process to form $N 1$-acetyl- $N 2$-formyl-5-methoxykynuramine (AFMK) from melatonin, an important metabolite of Trp produced in the skin. Melatonin is a wellknown regulator of circadian rhythmicity and skin pigmentation, and also stimulates the expression of antioxidative enzymes and DNA repair and has immunomodulatory and antitumor properties [14]. There are 2 known isoforms of IDO, IDO1, and IDO2, although IDO2 is less characterized and its function remains unclear $[15,16]$.

Trp catabolism is known as a central pathway preserving the immunosuppressive TME in many types of cancers [17]. Tumor cells or myeloid cells in the TME or draining lymph nodes express high levels of IDO1, the first and rate-limiting enzyme in the degradation of Trp, which result in Trp depletion in the TME, followed by the inhibition of T-cell responses. T cells sense low Trp levels via uncharged tRNAs and subsequently activate the general control nonderepressible 2 (GCN2) kinase and induce amino acid starvation, which causes cell cycle arrest and cell death [17]. This results in local immunosuppression in the TME. Trp degradation by TDO2, an alternative route of Trp degradation in tumors, creates Trp depletion together with IDO1, which is completed by tumor cells and myeloid cells [17]. Trp catabolism by IDO1 and TDO2 results in Trp depletion and accumulation of Trp metabolites.

KYN binds AhR as an intracellular transcription factor, which plays roles in the autoimmune and tumor immunity process. Previous studies suggest that the binding of KYN to AhR causes differentiation of CD4 $\mathrm{T}$ cells into Treg cells and inhibits its differentiation into interleukin 17-producing Th17 cells [18]. In addition, the ligand-activated AhR also induces the activation of IDO and promotes 
Table 1 List of antibodies and positive controls used for immunohistochemistry

\begin{tabular}{lllll}
\hline Antibodies & Host and type & Source & Dilution ratio & Positive controls \\
\hline IDO1 & Mouse monoclonal & Origene (Rockville, MD) & $1: 100$ & Lymph node \\
TDO2 & Mouse monoclonal & LifeSpan BioSciences (Seattle, WA) & $1: 100$ & Adrenal gland \\
AhR & Mouse monoclonal & Santa Cruz Biotechnology (Santa Cruz, CA) & $1: 100$ & Duodenum \\
\hline
\end{tabular}

the transcription of immunosuppressive mediators, such as interleukin 10 and prostaglandin E2 in DCs [19]. Therefore, the activation of AhR by the IDO1/TDO2 product KYN leads to the generation of immunotolerant DCs and Treg and affects the TME by decreasing its ability to recognize and eradicate cancer cells [12].

IDO is activated in tumor, stromal, and innate immune cells in various cancers, and its expression is correlated with a less favorable prognosis [20]. Increased TDO2 expression is associated with a higher grade, estrogen receptor-negative status, and a shorter overall survival (OS) in triple-negative breast cancer [21], whereas AhR overexpression in several cancers can be a positive or negative prognostic factor, depending on the type of cancer [22]. High AhR expression in breast cancer correlates with higher expression of several genes in inflammation, endogenous Trp metabolism, and the invasion signaling pathway [23].

The IDO1/TDO2-AhR pathway is now an important target for the development of novel cancer immunotherapy [12]. However, to the best of our knowledge, there has been no study on the expressions of IDO1, TDO2, and AhR in MCCs. Therefore, in this study, we investigated IDO1, TDO2, and AhR expressions in tumor cells and TME stromal cells in MCPyV-positive and MCPyV-negative MCCs. We also evaluated the relationship of these markers with clinicopathological factors and the prognosis of MCC.

\section{Materials and methods}

\subsection{Samples}

In this study, 43 formalin-fixed, paraffin-embedded samples were prepared. These included $24 \mathrm{MCPyV}$-positive MCCs (15 samples from the United Kingdom and 9 samples from Japan) and 19 MCPyV-negative MCCs (14 samples from the United Kingdom and 5 from Japan) samples. The MCPyV-negative MCC samples contained 12 MCC samples combined with squamous cell carcinoma (SqCC) or Bowen

Table 2 Comparison of clinicopathological parameters of MCCs based on MCPyV status

\begin{tabular}{|c|c|c|c|}
\hline Clinicopathological parameters & MCPyV-positive & MCPyV-negative & $P$ \\
\hline \multicolumn{4}{|l|}{ Sex, n $(\%)$} \\
\hline Male & $5(20.8)$ & $6(31.6)$ & \multirow[t]{2}{*}{.43} \\
\hline Female & $19(79.2)$ & $13(68.4)$ & \\
\hline Age $(y)$, mean \pm SD & $77.45 \pm 10.34$ & $84.68 \pm 9.63$ & $.021 *$ \\
\hline \multicolumn{4}{|l|}{ Race, n (\%) } \\
\hline Japanese & $9(37.5)$ & $5(26.3)$ & \multirow[t]{2}{*}{.442} \\
\hline UK White & $15(62.5)$ & $14(73.7)$ & \\
\hline \multicolumn{4}{|l|}{ Staging (I/II or III/IV), n (\%) } \\
\hline $\mathrm{I} / \mathrm{II}$ & $23(95.8)$ & $15(78.9)$ & \multirow[t]{2}{*}{.09} \\
\hline III/IV & $1(4.2)$ & $4(21.1)$ & \\
\hline \multicolumn{4}{|l|}{ Radical excision (no/yes), n (\%) } \\
\hline No & $3(13)$ & $6(37.5)$ & \multirow[t]{2}{*}{.078} \\
\hline Yes & $20(87)$ & $10(62.5)$ & \\
\hline IDO1 $\mathrm{H}$-score in MCC tumor cells, mean $\pm \mathrm{SD}$ & $28.96 \pm 31.86$ & $94.32 \pm 39.49$ & $<.001 *$ \\
\hline $\mathrm{TDO} 2 \mathrm{H}$-score in MCC tumor cells, mean $\pm \mathrm{SD}$ & $97.4 \pm 39.90$ & $106.32 \pm 49.49$ & .65 \\
\hline AhR H-score in MCC Tumor Cells, Mean \pm SD & $76.29 \pm 50.01$ & $71.42 \pm 33.70$ & .932 \\
\hline IDO1 expressed by TME $(\%)^{\text {a }}$ of MCCs, mean \pm SD & $46.25 \pm 23.56$ & $39.67 \pm 24.38$ & .444 \\
\hline TDO 2 expressed by TME $(\%)$ of MCCs, mean \pm SD & $48.13 \pm 18.29$ & $82.95 \pm 14.49$ & $<.001 *$ \\
\hline AhR expressed by TME (\%) of MCCs, mean \pm SD & $88.38 \pm 12.80$ & $95.11 \pm 7.49$ & .054 \\
\hline
\end{tabular}


disease. A summary of clinicopathological data is listed in Supplementary Table S1. This study was approved by the institutional review board of Medical Faculty, Tottori University, Japan.

\subsection{Immunohistochemistry}

Formalin-fixed, paraffin-embedded samples were sectioned into $4-\mu \mathrm{m}$-thick pieces, followed by deparaffinization and rehydration. Antigen retrieval was performed by incubating the sections for 40 minutes at $100^{\circ} \mathrm{C}$ in Nichirei Heat Pro II (Tokyo, Japan). After blocking endogenous peroxidase activity for 5 minutes, sections were incubated for 60 minutes with the first antibody and then incubated with the secondary antibody for 30 minutes. Sections were incubated with diaminobenzidine for $10 \mathrm{mi}-$ nutes; all these processes used the Nichirei Histo Stainer. After washing the sections using phosphate-buffered saline, they were counterstained with hematoxylin for $5 \mathrm{sec}-$ onds, and then rehydrated and mounted. The primary antibodies (IDO1, TDO2, AhR) used in this study and the tissues used as positive controls are listed in Table 1. The stained tissue slides were evaluated by pathologists and researchers who were blinded to the patients' clinical data. The cytoplasm and/or nucleus of IDO1-, TDO2-, and AhRpositive cells were stained, and the stained tumor cells of MCC and combined tumor were evaluated using the modified $\mathrm{H}$-score. The percentage of staining was summed and multiplied by values according to the intensity level $(0$, not stained; 1 , weakly stained; 2 , moderately stained; and 3 , strongly stained), and the $\mathrm{H}$-score ranged from 0 to a maximum of 300 [24]. The frequency of immunoreactive stromal cells in the TME of MCC included fibroblasts, endothelial cells, and inflammatory cells, which were stained by immunohistochemical staining, and were recorded as a percentage of immunoreactive cells in the all-component stromal cells in the TME of MCC. We evaluated immunohistochemically all cells in the areas of TME, which consist of the intratumoral stroma and surrounding morphologically reactive stroma. Entrapped skin appendages and the surrounding normal tissues as well as ulcerated tumor lesions were excluded. The internal negative control used nonneoplastic skin and subcutaneous tissues from MCC samples.

\subsection{Statistical analysis}

All clinicopathological parameters, such as age, sex, race, and immunohistochemistry results, were differentiated based on the MCPyV status and analyzed using the Mann-Whitney $U$ test. The survival analysis was evaluated using the Kaplan-Meier method in accordance with IDO1/ TDO2/AhR expressions. The significant differences were examined using the log-rank test. The Cox proportional hazards regression model was applied to perform univariate and multivariate analyses, and those variables that achieved statistical significance in the univariate analysis were included in the multivariable analysis. All data were analyzed statistically using SPSS software (version 21.0 J; SPSS Japan, Tokyo, Japan), and a $P$ value of less than .05 was considered statistically significant.

\section{Results}

\subsection{Characteristics of clinical background}

The 43 cases of MCC used in this study, comprising 24 MCPyV-positive MCCs and 19 MCPyV-negative MCCs, are described in Table 2 and Supplementary Table S1. There was a significant difference in the ages of both groups: MCPyV-negative MCC cases (mean, 84.68 years) were older than MCPyV-positive cases (mean, 77.45 years), as shown in Table $2(P=.021)$. The other clinical parameters, such as sex, race, and staging, were not different in the 2 groups.

\subsection{Histologic findings}

Immunohistochemical staining was performed to evaluate the Trp catabolism process through the KYN pathway (IDO1, TDO2, and AhR) in MCC tumor cells and the surrounding TME cells, and the results are summarized in Table 2 and Supplementary Tables S2, S3, and S4. The representative immunostaining features of IDO1, TDO2, and AhR in MCPyV-positive and MCPyV-negative MCC cases are shown in Fig. 1. The H-score of IDO1 was significantly lower in MCPyV-positive MCC than in MCPyV-negative MCC (Fig. 1E and F, Table 2; $P<.001$ ), and there was no difference in IDO1 expression in the TME of both groups. TDO2 was significantly more frequent in the TME of MCPyV-negative MCC than in that of MCPyV-positive MCC (Fig. 1H and G, Table $2 ; P<.001)$. Conversely, the $\mathrm{H}$-score of TDO2 expression in tumor cells of these subgroups was not significantly different. The similar trend results as TDO2 expression were seen in AhR evaluation. AhR expression was more frequent in the TME of MCPyV-negative cases than in MCPyV-positive cases (Fig. 1J and I), although the trend was not statistically significant $(P=.054)$, whereas MCPyV-positive and MCPyV-negative MCC tumor cells showed similar H-scores of their expression.

In combined MCC and SqCC cases, tumor cells in the MCC component showed a significantly higher H-score of IDO1 expression than did those in the SqCC cases (Supplementary Table S4; $P<.001$ ). TDO2 expression of tumor cells in the MCC component was significantly higher than that in the SqCC component in combined MCC and SqCC cases (Supplementary Table S4; $P=.04$ ). AhR expression was higher in SqCC components than in MCC components, but not statistically significant (Supplementary Table S4). 


\section{MCPyV-positive MCC}

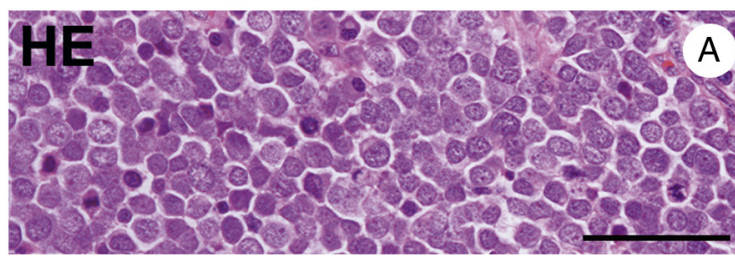

MCPYV-LT (CM2B4)
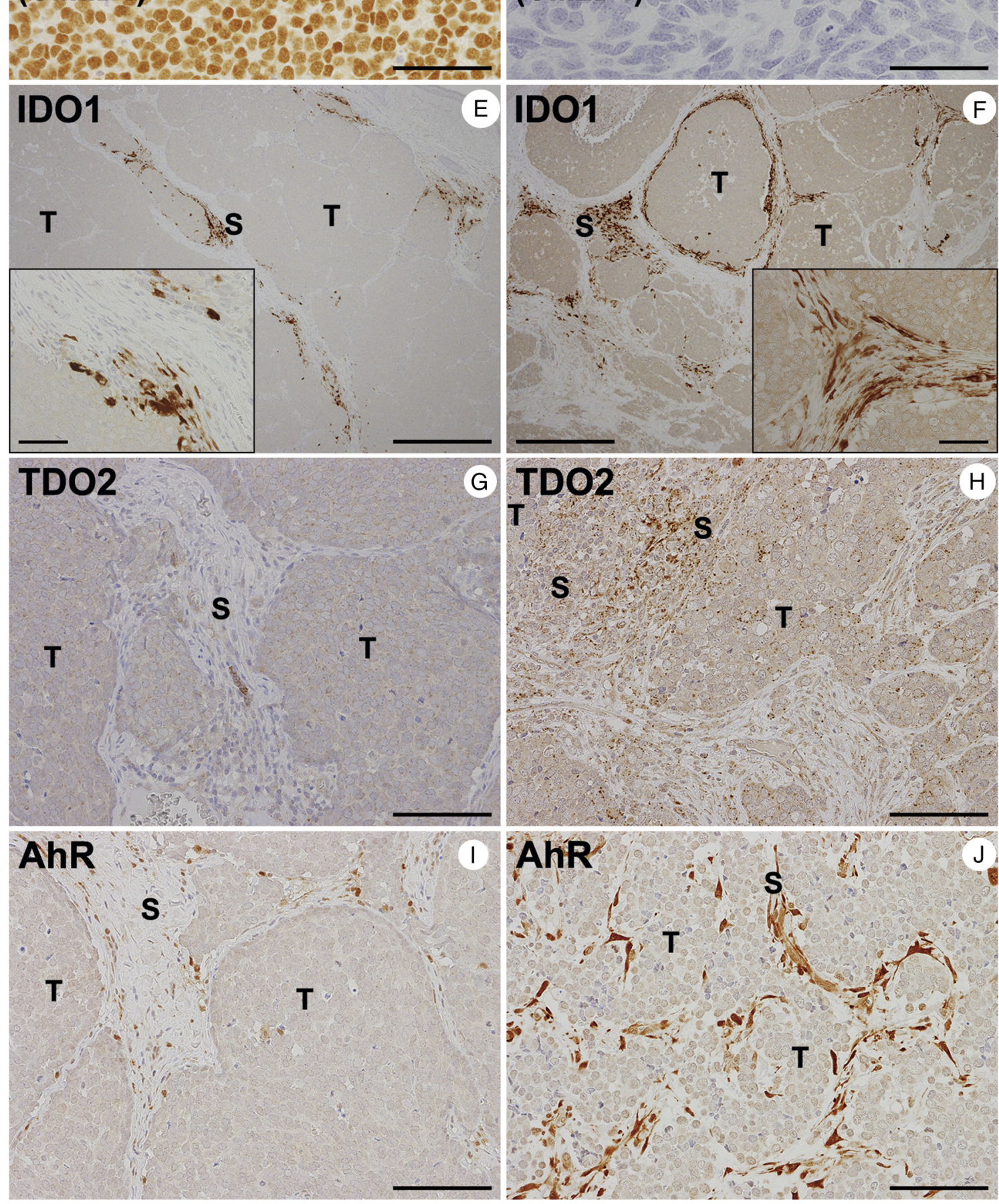

\section{MCPyV-negative MCC}

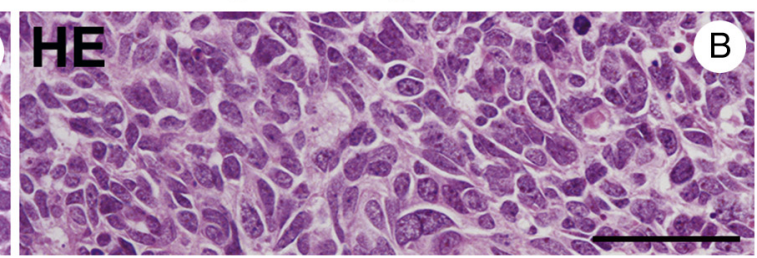

(CM2B4)
MCPyV-LT
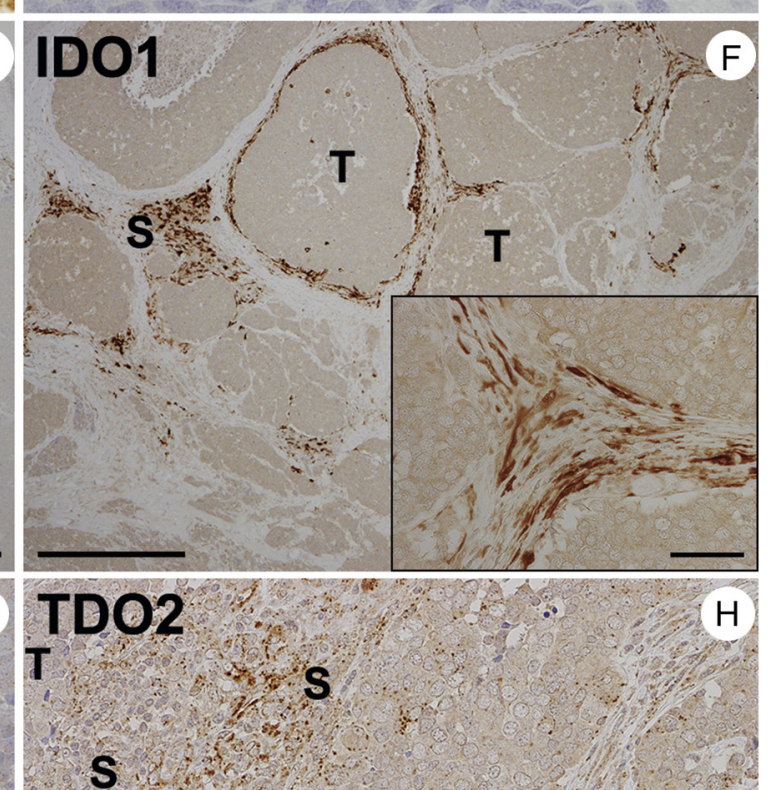

$\mathbf{T}$

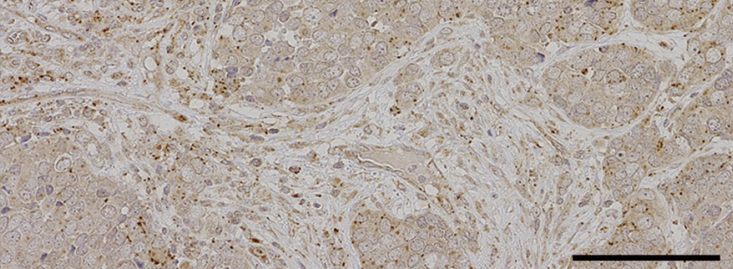




\subsection{Prognostic analysis}

The Kaplan-Meier with log-rank test was used to analyze prognostic survival in this study, and the results are summarized in Table 3. A lower-than-mean IDO1 expression in MCC tumor cells $(\mathrm{H}$-score $<58)$ corresponded with a favorable OS (Fig. 2A; $P=.043$ ) but not disease-specific survival (DSS; Fig. 2B; $P=.119)$. A higher-than-mean TDO2 expression compared with the TDO2 low expression group in TME stromal cells of MCCs $(\geq 64 \%)$ related not only to poor OS (Fig. $2 \mathrm{C} ; P=.008$ ) but also to poor DSS (Fig. 2D; $P=.016$ ). As well as to TDO2 expression in the TME of MCCs, higher AhR expression ( $\geq 91 \%$ ) exhibited a shorter OS (Fig. 2E; $P=.035$ ), which could not be seen in DSS (Fig. 2F; $P=.291$ ).

Clinicopathological parameters and IDO1, TDO2, and AhR expressions in both tumor cells and TME stromal cells for the prediction of OS and DSS were further investigated by univariate and multivariate analyses with the Cox regression model. The results are shown in Table 4. Results from the univariate analysis indicated that the negativity of the MCPyV state (coefficient, 1.422; hazard ratio [HR], 4.145; $P=.004$ ) and advanced age (coefficient, 2.075; HR, 7.968; $P=.045$ ) were unfavorable factors for OS. Similarly, with the MCPyV-negative state and advanced age, higher expression of TDO2 in TME stromal cells (coefficient, 1.234; HR, 3.437; $P=.013$ ) and AhR in TME stromal cells (coefficient, 1.061; HR, $2.889 ; P=.044)$ also shortened OS, and radical excision treatment (coefficient, -1.179 ; HR, $0.308 ; P=.02$ ) could extend OS. Higher TDO2 expression in TME stromal cells (coefficient, 2.158; HR, 8.653; $P=.045$ ) reduced DSS, and radical excision treatment (coefficient, $-2.048 ; \mathrm{HR}, 0.129 ; P=.006$ ) could lengthen DSS. Then, 5 variables that influenced OS were included in a multivariate Cox proportional hazards analysis, and no variables showed a prognostic significance to OS, whereas only radical excision treatment showed a significant increase in DSS (coefficient, -2.076 ; HR, 0.125; $P=.033$ ).

\section{Discussion}

$\mathrm{MCPyV}$ is known to play roles in the carcinogenesis of most MCC cases, namely, MCPyV-positive MCC. MCPyV- negative MCC shows a high frequency of DNA mutations associated with UV damage, disruption of RB1 and TP53, the presence of a high degree of aneuploidy, and mutations in genes related to responses to DNA impairment and repair. $\mathrm{MCPyV}$-positive MCC usually has few somatic mutations and little evidence of UV damage, and most MCPyV-positive cases contain intact $R B 1$ and wild-type TP53 [25]. In our previous study, we showed that MCPyV-positive MCC tumor cells had uniform round nuclei and less cytoplasm, whereas MCPyV-negative tumor cells had irregular nuclei and abundant cytoplasm [4]. The MCPyV status relates to different survival and prognosis. Patients with MCPyV-positive MCC usually have longer survival and better prognosis compared with those with MCPyV-negative MCC $[7,8]$. In this study, we reconfirmed the results of our previous study that patients with MCPyV-negative MCC have unfavorable length of survival $[5,24,26]$, which is worse if they are elderly [24].

The IDO1/TDO2-AhR pathway has been known to play roles in tumorigenesis by creating an immunosuppressive environment through interactions among tumor cells and stromal cells in the TME, which is beneficial for tumor progression. Therefore, new immuno-oncology therapies to target this signaling pathway have been developed [12]. We first evaluated the expression status of the IDO1/TDO2-AhR pathway in tumor cells and TME stromal cells of MCC to help develop new immunotherapies for patients with MCC.

IDO1 as the first key rate-limiting enzyme in Trp catabolism is ubiquitously expressed by many kinds of tissues and cells that constitute the TME, including endothelial cells, macrophages, and fibroblasts [12,27]. In addition, DCs and myeloid-derived suppressor cells in the TME are coerced by cancer cells to express IDO1, resulting in avoiding immunosurveillance [12]. IDO1 expression by cancer cells is also seen in acute myeloid leukemia, breast, cervical, colorectal, and endometrial cancers, and glioma. Its overexpression has usually been related to negative prognostic factors and worse outcome measures $[20,28,29]$. Trott et al [30] reported that IDO1 was expressed by tumor cells not only in renal cell carcinoma but also in the TME. IDO1 expression scores in both tumor cells and interstitial cells of renal cell carcinoma were higher than those in normal kidney tissue.

In our study, tumor cells in MCPyV-negative MCCs express significantly higher IDO1 compared with MCPyV-

\footnotetext{
Fig. 1 Representative images of immunohistochemical staining of MCPyV-positive and MCPyV-negative MCC. The morphology and immunostaining of MCPyV-positive MCC (A, C, E, G, and I) and MCPyV-negative MCC (B, D, F, H, and J) are shown. MCPyV-positive MCC tumor cells (A) had nuclei with a regular shape and less cytoplasm than MCPyV-negative MCC cells (B). The positivity of MCPyV-LT was shown as a dense diffuse nuclear reactivity in all MCPyV-positive MCCs (C), but not in MCPyV-negative MCCs (D). A-D, The bar represents $50 \mu \mathrm{m}$. IDO1 was expressed more strongly in MCPyV-negative tumor cells (F) than in MCPyV-positive tumor cells (E; H-score: mean $\pm \mathrm{SD}, 94.32 \pm 39.49$ and $28.96 \pm 31.86$, respectively; $P<.001$; bar, $500 \mu \mathrm{m}$ ), whereas its expression in TME cells was not different in both MCC subgroups. Insets, The bar represents $50 \mu \mathrm{m}$. The expressions of TDO2 and AhR in MCC tumor cells were similar in MCPyV-positive (G and I, respectively; bar, $100 \mu \mathrm{m})$ and MCPyV-negative (H and J, respectively; bar, $100 \mu \mathrm{m}$ ) tumor cells. In TME stromal cells, TDO2 was expressed more frequently in MCPyV-negative MCC than in MCPyV-positive MCC (mean \pm SD, $82.95 \% \pm 14.49 \%$ versus $48.13 \% \pm 18.29 \%$; $P<.001$ ). A similar tendency was seen in AhR expressed in MCPyV-negative TME stromal cells, which was more prominent than that in MCPyV-positive TME stromal cells (mean $\pm \mathrm{SD}, 95.11 \% \pm 7.49 \%$ and $88.38 \% \pm 12.80 \%$, respectively; $P=.054$ ). A and B, Hematoxylin-eosin stain; C-J, immunostain. Abbreviations: T, tumor cells; $\mathrm{S}$, stromal cells in the TME.
} 
Table 3 Comparison between clinicopathological parameter including immunohistochemistry (H-score) and OS or DSS

\begin{tabular}{lrll}
\hline Factor & Mean & OS $(P$ value $)$ & DSS $(P$ value $)$ \\
\hline IDO1 in MCC H-score $<58 / \geq 58$ & 57.84 & $.043 *$ & .119 \\
TDO2 in MCC H-score $<101 / \geq 101$ & 101.34 & .177 & .087 \\
AhR in MCC H-score $<74 / \geq 74$ & 74.14 & .188 & .813 \\
IDO1 expressed by TME $(\%)^{\mathrm{a}}<43 / \geq 43$ & 43.43 & .279 & .931 \\
TDO2 expressed by TME $(\%)<64 / \geq 64$ & 63.51 & $.008 *$ & $.016 *$ \\
AhR expressed by TME $(\%)<91 / \geq 91$ & 91.35 & $.035 *$ & .291 \\
MCPyV-Positive/Negative & & $.002 *$ & .059 \\
Age $(<75 / \geq 75$ y) & & $.017 *$ & .554 \\
Sex (male/female) & & .559 & .68 \\
Race $($ Japanese/UK White) & & .649 & .742 \\
Staging (I/II or III/IV) & & $.014 *$ & .894 \\
Radical excision $($ No/Yes) & & $.001 *$ \\
\hline
\end{tabular}

Abbreviations: AhR, aryl hydrocarbon receptor; DSS, disease-specific survival; IDO1, indoleamine 2,3-dioxygenase 1; MCC, Merkel cell carcinoma; MCPyV, Merkel cell polyomavirus; OS, overall survival; TDO2, tryptophan 2,3-dioxygenase2.

a TME (\%), immunoreactive cell frequency (\%) in all component stromal cells of TME of MCCs including fibroblasts, vessels, and inflammatory cells.

* Statistically significant (Kaplan-Meier method; $P<.05$ ).

positive tumor cells. A higher-than-total-mean IDO1 expression of tumor cells (H-score $\geq 58$ [total mean]) is associated with significantly worse OS than the lower-than-total-mean
IDO1 expression ( $\mathrm{H}$-score $<58$ ), but there is no relationship with DSS. This study shows that there is no difference in IDO1 expression by TME stromal cells between MCPyV-
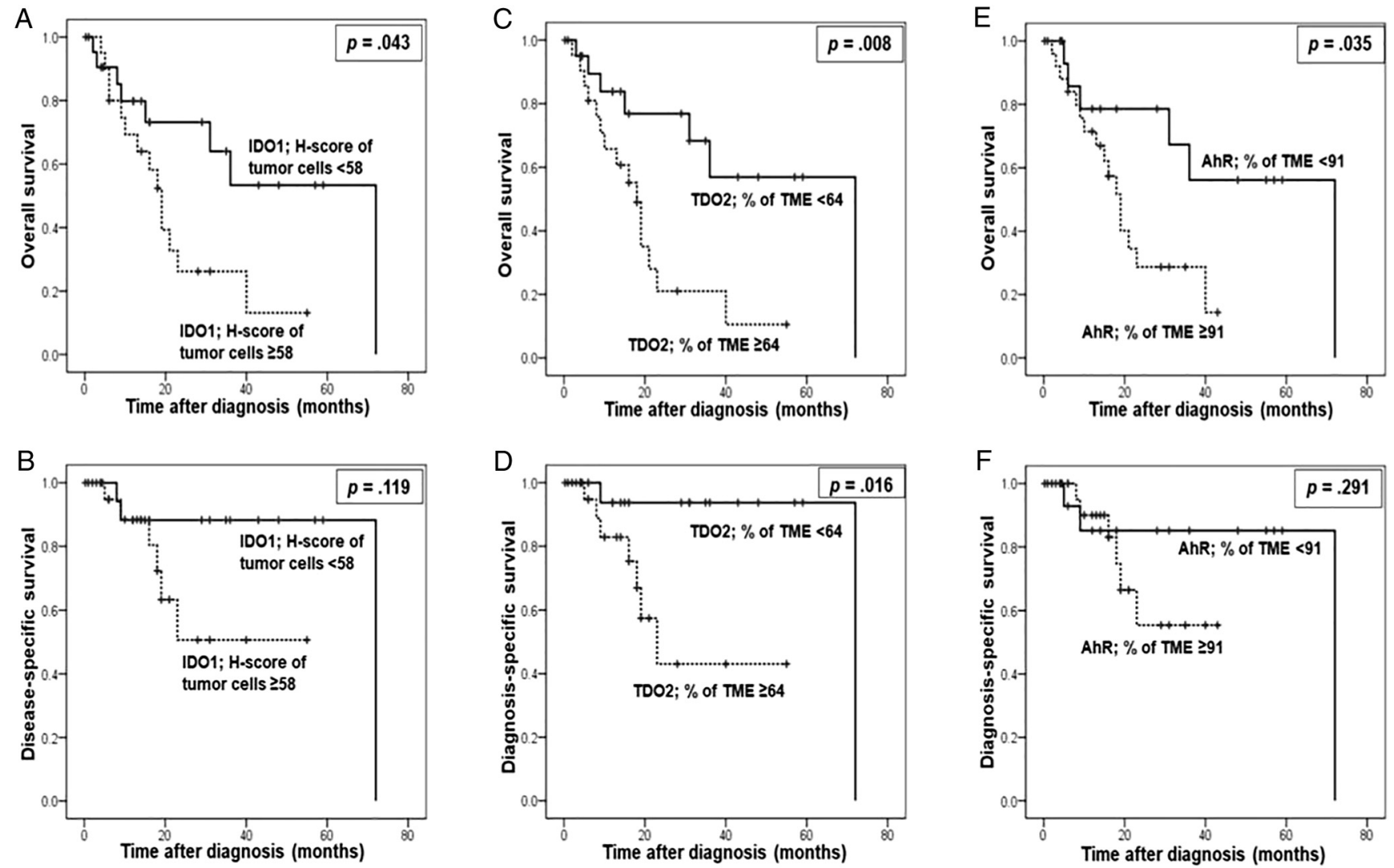

Fig. 2 OS (A, C, and E) and MCC-specific survival (DSS; B, D, and F) classified by mean expression status of IDO1 in MCC tumor cells (A and $\mathrm{B})$, TDO2 in the TME of MCC (C and D), and AhR in the TME of MCC (E and F). Kaplan-Meier with log-rank test evaluated the statistical significance. A and B, Patients with a lower-than-mean IDO1 expression in MCC tumor cells $(\mathrm{H}$-score $<58)$ survived significantly longer than did those with higher IDO1 expression (H-score $\geq 58$ ) in OS (A, $P=.043)$, but there was no significant difference in DSS (B, $P=.119)$. C and D, Patients with a lower-than-mean TDO2 expression in the TME $(<64 \%)$ had a significantly longer survival than did those with a higher TDO2 expression in the TME ( $\geq 64 \%$; OS: $P=.008[\mathrm{C}]$ and DSS: $P=.016[\mathrm{D}])$. E and F, Patients with a lower AhR expression in the TME $(<91 \%)$ had a significantly longer survival than did those with a higher AhR expression in the TME $(\geq 91 \%)$ in OS (E, $P=.035)$, whereas there was no significant difference in DSS (F, $P=.291)$. 
Table 4 Univariate and multivariate Cox proportional hazard regression analysis of prognostic factors for mortality in MCC cases

\begin{tabular}{|c|c|c|c|c|c|c|c|c|}
\hline \multirow[t]{2}{*}{ Factor } & \multicolumn{4}{|l|}{ OS } & \multicolumn{4}{|l|}{ DSS } \\
\hline & Coefficient & HR & $95 \% \mathrm{CI}$ & $P$ & Coefficient & HR & $95 \% \mathrm{Cl}$ & $P$ \\
\hline \multicolumn{9}{|l|}{ Univariate } \\
\hline MCPyV-positive/negative & 1.422 & 4.145 & $1.556-11.04$ & $.004 *$ & 1.428 & 4.169 & $0.833-20.868$ & .082 \\
\hline Sex (male/female) & 0.29 & 1.336 & $0.508-3.513$ & .557 & 0.335 & 1.398 & $0.28-6.982$ & .683 \\
\hline Age $(<75 / \geq 75$ y) & 2.075 & 7.968 & $1.052-60.375$ & $.045 *$ & 1.013 & 2.753 & $0.336-22.59$ & .346 \\
\hline Race (Japanese/UK White) & -0.31 & 0.734 & $0.256-2.098$ & .563 & -0.271 & 0.763 & $0.15-3.878$ & .744 \\
\hline Staging (I/II or III/IV) & 0.283 & 1.327 & $0.388-4.544$ & .652 & 0.142 & 1.152 & $0.141-9.407$ & .895 \\
\hline Radical excision (no/yes) & -1.179 & 0.308 & $0.114-0.829$ & $.02 *$ & -2.048 & 0.129 & $0.03-0.562$ & $.006 *$ \\
\hline IDO1 in MCC H-score $<58 / \geq 58$ & 0.915 & 2.497 & $0.993-6.279$ & .052 & 1.202 & 3.326 & $0.666-16.597$ & .143 \\
\hline TDO2 in MCC H-score $<101 / \geq 101$ & 0.586 & 1.797 & $0.755-4.275$ & .185 & 1.188 & 3.28 & $0.774-13.907$ & .107 \\
\hline AhR in MCC H-score $<74 / \geq 74$ & 0.574 & 1.776 & $0.743-4.244$ & .197 & -0.173 & 0.841 & $0.198-3.567$ & .814 \\
\hline IDO1 expressed by $\operatorname{TME}^{\mathrm{a}}(\%)<43 / \geq 43$ & -0.48 & 0.619 & $0.256-1.497$ & .287 & -0.061 & 0.941 & $0.235-3.774$ & .931 \\
\hline TDO2 expressed by TME $(\%)<64 / \geq 64$ & 1.234 & 3.437 & $1.302-9.069$ & $.013 *$ & 2.158 & 8.653 & $1.049-71.413$ & $.045 *$ \\
\hline AhR expressed by TME $(\%)<91 / \geq 91$ & 1.061 & 2.889 & $1.03-8.1$ & $.044 *$ & 0.842 & 2.32 & $0.463-11.625$ & .306 \\
\hline \multicolumn{9}{|l|}{ Multivariate } \\
\hline MCPyV-positive/negative & 0.111 & 1.117 & $0.204-6.115$ & .898 & -0.427 & 0.652 & $0.031-13.69$ & .783 \\
\hline Age $(<75 / \geq 75$ y) & 1.949 & 7.021 & $0.804-61.313$ & .078 & 0.863 & 2.37 & $0.195-28.777$ & .498 \\
\hline Radical excision (no/yes) & -0.769 & 0.463 & $0.146-1.467$ & .191 & -2.076 & 0.125 & 0.019-0.847 & $.033 *$ \\
\hline TDO2 expressed by TME $(\%)<64 / \geq 64$ & 1.331 & 3.785 & $0.883-16.229$ & .073 & 1.962 & 7.115 & $0.499-101.439$ & .148 \\
\hline AhR expressed by TME $(\%)<91 / \geq 91$ & -0.037 & 0.964 & $0.205-4.532$ & .963 & 0.009 & 1.009 & $0.071-14.246$ & .995 \\
\hline
\end{tabular}

positive and MCPyV-negative MCC. These results suggest that the MCPyV status may be related to differential IDO1 expression only in MCC tumor cells.

On the other hand, TDO2, expressed mainly by the liver to maintain the systemic homeostasis of Trp through dietary intake metabolism [12,31], is expressed by several cancers such as glioma, hepatocellular carcinoma, bladder cancer, and melanoma [31,32]. Chen et al [33] showed that both IDO1 and TDO2 expressions were significantly increased in colorectal cancer tissues and strongly associated with lymph node metastasis and advanced stage of cancer.

We see a slightly higher tendency for TDO2 expression in MCPyV-negative tumor cells than in positive cells, but the difference is not statistically significant. On the contrary, TDO2 expression in TME stromal cells of MCPyV-negative MCC is significantly higher than that of MCPyV-positive MCC. Patients with a higher mean TDO2 expression in TME stromal cells of MCC $(\geq 64 \%)$ are associated with a significantly more unfavorable OS and DSS compared with those with a lower mean TDO2 expression $(<64 \%)$. Higher TDO2 expression in stromal cells in the TME of MCC is associated with a poor prognosis, but expression in tumor cells is not.

Numerous types of cancer cells preferentially express IDO1 or TDO2 and express both in some cases [12]. In most MCCs, both IDO1 and TDO2 are expressed in tumor cells and the TME.

$\mathrm{KYN}$ is an endogenous ligand of AhR, the ligand-activated transcription factor. Their binding (KYN-AhR complex) induces a tolerogenic immune response by inducing Treg proliferation and immunotolerant DCs [18]. AhR activation collectively fosters an immunosuppressive TME that is defective in recognizing and eradicating cancer cells [12]. In addition, AHR overexpression can affect prognosis positively or negatively. It relies on the type of cancer and the endogenous role of a receptor, because AhR can exhibit tumor-specific prooncogenic and tumor suppressor-like functions [22]. Cancer cells can use the KYN-AhR complex for their benefit, as it promotes a pro-tumorigenic effect that increases cell motility [31,34]. TDO2-expressed triple-negative breast cancer lines produced Trp metabolites to activate AhR, resulting in TDO2 induction, and AhR also accelerated migration in an AhR-dependent fashion [34]. The immunohistochemistry of $\mathrm{AhR}$ in breast cancers revealed the presence of $\mathrm{AhR}$ in tumor cells and in the intratumoral nonepithelial tissue (endothelial cells and immune cells including lymphocytes) [23].

We showed that AhR expressed by the TME in MCPyVnegative MCC is higher than that in MCPyV-positive MCC, although it is not statistically significant $(P=.054)$, whereas tumor cells in MCPyV-positive and MCPyV-negative MCC expressed similar levels of AhR. A higher mean of AhR expression ( $\geq 91 \%$ ) in the TME of MCC is correlated with a significantly shortened OS. These results suggest that higher AhR expression in the TME may be associated with MCPyV negativity and poor prognosis in MCC, although AhR expression is not different in tumor cells between MCPyV-positive and MCPyV-negative MCC. This may be partially caused in MCC by the known mechanism of AhR as a negative prognostic factor, whereas Trp metabolites can activate AhR to induce an immunosuppressive TME leading to tumor growth. 
Tables 2 and 3 show that there were significant differences between MCPyV-negative and MCPyV-positive cases in the expression of IDO1 in tumor cells and TDO2 in TME, and that higher means of IDO1 in tumor cells, TDO2 in TME, and AhR in TME were associated with unfavorable prognosis. Therefore, we also evaluated the correlation among these 3 variables, and there was a significant strong positive correlation between IDO1 in tumor cells and TDO2 in TME, whereas IDO1 in tumor cells with AhR in TME and TDO2 in TME with AhR in TME showed weak positive correlations and were not statistically significant (unpublished data). Higher mean of TDO2 expression in TME as shown in Tables 3 and 4 influenced patients' prognosis with shorter survival in OS and DSS, whereas higher mean of IDO1 in tumor cells only affected OS as shown in Table 3. This suggests that stromal cell involvement by expressing TDO2 is a more important factor for the tumorigenesis and biology of MCC than IDO1 expressed by tumor cells, although both of them will give an impact on prognosis of MCC.

The IDO1/TDO2-AhR pathway has become a focus of attention because tumorigenesis is influenced by immunological responses in the TME, which lead to tumor growth. Therefore, to activate the immune system to kill cancer cells, several approaches targeting the IDO1/TDO2-KYN-AhR signaling circuitry have been tried: (1) developing IDO1/TDO2 inhibitors to prevent KYN production as a ligand for AhR, (2) systemic depletion of KYN by engineered kynureninase, and (3) inhibition of AhR activation by synthetic AhR modulators [12]. Interactions between $\mathrm{AhR}$ and its ligand increase immunosuppression leading to tumor growth; therefore, a synthetic AhR antagonist has been created, but it has not yet been fully investigated [12]. The lack of anticancer activity of IDO1 inhibitors (epacadostat and indoximod) as monotherapy has led to a new approach of using IDO1 inhibitors in combination with approved anticancer drugs. Several selective IDO1 inhibitors have entered the clinical trial phase as a combined therapy in cancers such as melanoma, head and neck cancer, colorectal cancer, or solid tumors. Their effectiveness is still under evaluation. Epacadostat has shown some promising results in melanoma when used with an immune-checkpoint inhibitor (PD-1 inhibitor, pembrolizumab) [12].

PD-L1 and IDO1 were expressed by melanoma cells based on morphology but could also be expressed by some stromal cells [35]. Interferon- $\gamma$ is a hallmark tumoricidal cytokine secreted from CTL, and it up-regulates IDO1 and PD-L1, which attenuate the cytotoxicity of CTLs. Melanoma develops resistance to anticytotoxic T-lymphocyte associated protein 4 (CTLA-4) therapy by up-regulation of IDO1 [12].

The PD1-PD-L1 immune-checkpoint pathway is a key therapeutic target in reactivating immune responses against various types of cancers, including MCC [25]. MCC has been identified as an immunogenic cancer because of the presence of immune response to MCPyV T antigens in the serum of patients with MCPyV-positive MCC and neoantigens associated with very frequent DNA mutations in MCPyV-negative MCC. Three clinical trials of immune-checkpoint inhibition using the anti-PD1 or anti-PD-L1 antibody in patients with advanced- stage MCC show higher and more durable response rates than conventional chemotherapy. However, a substantial fraction of advanced-stage MCC cases do not respond to these PD1 or PD-L1 blockade therapies [9-11]. Therefore, several clinical combination trials of immune-checkpoint inhibitors with CTLA-4 inhibitors, adoptive T-cell or natural killer cell transfers are underway in MCC. It is noteworthy that the response to immune-checkpoint blockade therapy was independent of the MCPyV or PD-L1 expression status [25].

In MCC, combination therapies of inhibitors targeting the IDO1/TDO2-KYN-AhR signaling circuitry with immunecheckpoint blockades against PD1, PD-L1, or CTLA-4 will be new clinical trials in the future to overcome the immune escape mechanism. Understanding and evaluating the immune escape mechanisms of the IDO1/TDO2-KYN-AhR signaling circuitry and immune-checkpoints in MCC is necessary to select patients who can benefit most from these immunotherapies.

In conclusion, we have shown that $\mathrm{MCPyV}$-negative $\mathrm{MCC}$ is significantly related to higher IDO1 expression in tumor cells and higher TDO2 expression by the TME than MCPyV-positive MCC. Using Kaplan-Meier analysis, a higher mean of IDO1 expression by tumor cells is associated with a significantly shorter OS, and a higher mean of TDO2 expression by the TME is related to a shorter OS and DSS. A higher mean of AhR expression by the TME shows a significantly unfavorable OS. Univariate analysis reveals that MCPyV positivity, age $(<75$ years), radical excision, and lower expressions of TDO2 and AhR in the TME are significantly linked with a longer OS. Radical excision and lower expression of TDO2 in the TME are also significantly associated with favorable DSS, but these are not statistically significant on multivariate analysis, except for radical excision that is significantly related to DSS. These results suggest that the IDO1/TDO2-AhR pathway may play a role in MCC tumorigenesis by inducing an immunosuppressive environment, which leads to tumor growth in MCC and influences the outcome. Therefore, it is important to evaluate the expression levels of the IDO1/TDO2-AhR pathway in both tumor cells and the TME of MCC to develop novel cancer immunotherapy and select the most likely patients to benefit from targeted therapies against the IDO1/TDO2-AhR pathway.

\section{Supplementary data}

Supplementary data to this article can be found online at https://doi.org/10.1016/j.humpath.2018.09.003.

\section{Acknowledgments}

We thank the laboratory staff at the Division of Molecular Pathology, Department of Pathology, Tottori University, Faculty of Medicine, particularly Mr H. Sugihara, for the skillful preparation of tissue slides. We would like to thank Enago (www.enago.jp) for the English language review. 


\section{References}

[1] Kuwamoto S. Recent advances in the biology of Merkel cell carcinoma Huм Pathol 2011;42:1063-77. https://doi.org/10.1016/j.humpath.2011. 01.020 .

[2] Ascoli V, Minelli G, Kanieff M, Frova L, Conti S. Merkel cell carcinoma: a population-based study on mortality and the association with other cancers. Cancer Causes Control 2011;22:1521-7. https://doi.org/10.1007/s10552-011-9826-4.

[3] Feng H, Shuda M, Chang Y, Moore PS. Clonal integration of a polyomavirus in human Merkel cell carcinoma. Science 2008;319:1096-100. https://doi.org/10.1126/science.1152586.

[4] Kuwamoto S, Higaki H, Kanai K, et al. Association of Merkel cell polyomavirus infection with morphologic differences in Merkel cell carcinoma. Hum Pathol 2011;42:632-40. https://doi.org/10.1016/j.humpath.2010.09.011.

[5] Higaki-Mori H, Kuwamoto S, Iwasaki T, et al. Association of Merkel cell polyomavirus infection with clinicopathological differences in Merkel cell carcinoma. Hum PATHOL 2012;43:2282-91. https://doi.org/10.1016/ j.humpath.2012.04.002.

[6] Bhatia K, Goedert JJ, Modali R, Preiss L, Ayers LW. Immunological detection of viral large $\mathrm{T}$ antigen identifies a subset of Merkel cell carcinoma tumors with higher viral abundance and better clinical outcome. Int J Cancer 2010;127:1493-6. https://doi.org/10.1002/ijc.25136.

[7] Sihto H, Kukko H, Koljonen V, Sankila R, Böhling T, Joensuu H. Merkel cell polyomavirus infection, large $\mathrm{T}$ antigen, retinoblastoma protein and outcome in Merkel cell carcinoma. Clin Cancer Res 2011;17: 4806-13. https://doi.org/10.1158/1078-0432.CCR-10-3363.

[8] Moshiri AS, Doumani R, Yelistratova L, et al. Polyomavirus-negative Merkel cell carcinoma: a more aggressive subtype based on analysis of 282 cases using multimodal tumor virus detection. J Invest Dermatol 2017;137:819-27. https://doi.org/10.1016/j.jid.2016.10.028.

[9] Kaufman HL, Russel J, Hamid O, et al. Avelumab in patients with chemotherapy-refractory metastatic Merkel cell carcinoma: a multicentre, single-group, open-label, phase 2 trial. Lancet Oncol 2016;17:1374-85. https://doi.org/10.1016/S1470-2045(16)30364-3.

[10] Ngiem PT, Bhatia S, Lipson EJ, et al. PD-1 blockade with pembrolizumab in advanced merkel-cell carcinoma. N Engl J Med 2016;374: 2542-52. https://doi.org/10.1056/NEJMoa1603702.

[11] Topalian SL, Bhatia S, Hollebecque A, et al. Non-comparative, open-label, multiple cohort, phase 1/2 study to evaluate nivolumab (NIVO) in patients with virus-associated tumors (checkmate 358): efficacy and safety in merkel cell carcinoma (MCC) (abstract). Cancer Res 2017;77: CT074. https://doi.org/10.1158/1538-7445.AM2017-CT074.

[12] Cheong JE, Sun L. Targeting the IDO1/TDO2-KYN-AhR pathway for cancer immunotherapy - challenges and opportunities. Trends Pharmacol Sci 2018;39:307-25. https://doi.org/10.1016/j.tips.2017.11.007.

[13] Cheong JE, Ekkati A, Sun L. A patent review of IDO1 inhibitors for cancer. Expert Opin Ther Pat 2018;28:317-30. https://doi.org/10.1080/ 13543776.2018.1441290

[14] Slominski AT, Hardeland R, Zmijewski MA, et al. Melatonin: a cutaneous perspective on its production, metabolism, and functions. J Invest Dermatol 2018;138:490-9. https://doi.org/10.1016/j.jid.2017.10.025.

[15] Ball HJ, Yuasa HJ, Austin CJD, Weiser S, Hunt NH. Indoleamine 2,3dioxygenase-2; a new enzyme in the kynurenine pathway. Int J Biochem Cell Biol 2009;41:467-71. https://doi.org/10.1016/j.biocel.2008.01.005.

[16] Ochs K, Ott M, Rauschenbach KJ, et al. Tryptophan-2,3-dioxygenase is regulated by prostaglandin $\mathrm{E} 2$ in malignant glioma via a positive signaling loop involving prostaglandin E receptor-4. J Neurochem 2016;136: 1142-54. https://doi.org/10.1111/jnc.13503

[17] Platten M, Doeberitz NVK, Oezen I, Wick W, Ochs K. Cancer immunotherapy by targeting IDO1/TDO and their downstream effectors. Front Immunol 2014;5:673. https://doi.org/10.3389/fimmu.2014.00673.

[18] Nguyen NT, Kimura A, Nakahama T, et al. Aryl hydrocarbon receptor negatively regulates dendritic cell immunogenicity via a kynurenine- dependent mechanism. Proc Natl Acad Sci U S A 2010;107:19961-6. https://doi.org/10.1073/pnas.1014465107.

[19] Vogel CFA, Goth SR, Dong B, Pessah IN, Matsumura F. Aryl hydrocarbon receptor signaling mediates expression of indoleamine 2,3dioxygenase. Biochem Biophys Res Commun 2008;375:331-5. https://doi.org/10.1016/j.bbrc.2008.07.156.

[20] Godin-Ethier J, Hanafi LA, Piccirillo CA, Lapointe R. Indoleamine 2,3dioxygenase expression in human cancers: clinical and immunologic perspectives. Clin Cancer Res 2011;17:6985-91. https://doi.org/10.1158/ 1078-0432.CCR-11-1331.

[21] D'Amato NC, Rogers TJ, Gordon MA, et al. A TDO2-AhR signaling axis facilitates anoikis resistance and metastasis in triple-negative breast cancer. Cancer Res 2015;75:4651-64. https://doi.org/10.1158/00085472.CAN-15-2011.

[22] Safe S, Cheng Y, Jin UH. The aryl hydrocarbon receptor (AhR) as a drug target for cancer chemotherapy. Curr Opin Toxicol 2017;2:24-9. https:// doi.org/10.1016/j.cotox.2017.01.012.

[23] Vacher S, Castagnet P, Chemlali W, et al. High AHR expression in breast tumors correlates with expression of genes from several signaling pathways namely inflammation and endogenous tryptophan metabolism. PLoS One 2018;13:e0190619. https://doi.org/ 10.1371/journal.pone.0190619.

[24] Iwasaki T, Matsushita M, Nonaka D, et al. Lower expression of CADM1 and higher expression of MAL in Merkel cell carcinomas are associated with Merkel cell polyomavirus infection and better prognosis. HuM PAтHоl 2016;48:1-8. https://doi.org/10.1016/j.humpath.2015.09.030.

[25] Becker JC, Stang A, DeCaprio JA, et al. Merkel cell carcinoma. Nat Rev Dis Primers 2017;3:17077. https://doi.org/10.1038/nrdp.2017.77.

[26] Kuromi T, Matsushita M, Iwasaki T, et al. Association of expression of the hedgehog signal with Merkel cell polyomavirus infection and prognosis of Merkel cell carcinoma. HuM PAтHOL 2017;69:8-14. https://doi.org/10.1016/j.humpath.2017.05.011.

[27] Munn DH, Mellor AL. IDO in the tumor microenvironment: inflammation, counter-regulation, and tolerance. Trends Immunol 2016;37: 193-207. https://doi.org/10.1016/j.it.2016.01.002.

[28] Brochez L, Chevolet I, Kruse V. The rationale of indoleamine 2,3-dioxygenase inhibition for cancer therapy. Eur J Cancer 2017;76:167-82. https://doi.org/10.1016/j.ejca.2017.01.011.

[29] Théate I, Baren NV, Pilotte L, et al. Extensive profiling of the expression of the indoleamine 2,3-dioxygenase 1 protein in normal and tumoral human tissues. Cancer Immunol Res 2015;3:161-72. https://doi.org/10.1158/2326-6066.CIR-14-0137.

[30] Trott JF, Kim J, Abu Aboud O, et al. Inhibiting tryptophan metabolism enhances interferon therapy in kidney cancer. Oncotarget 2016;7: 66540-57. https://doi.org/10.18632/oncotarget.11658.

[31] Opitz CA, Litzenburger UM, Sahm F, et al. An endogenous tumour-promoting ligand of the human aryl hydrocarbon receptor. Nature 2011;478: 197-203. https://doi.org/10.1038/nature10491.

[32] Pilotte L, Larrieu P, Stroobant V, et al. Reversal of tumoral immune resistance by inhibition of tryptophan 2,3-dioxygenase. Proc Natl Acad Sci U S A 2012;109:2497-502. https://doi.org/10.1073/pnas.1113873109.

[33] Chen IC, Lee KH, Hsu YH, Wang WR, Chen CM, Cheng YW. Expression pattern and clinicopathological relevance of the indoleamine 2,3dioxygenase 1/tryptophan 2,3-dioxygenase protein in colorectal cancer. Dis Markers 2016;2016:8169724. https://doi.org/10.1155/2016/ 8169724.

[34] Novikov O, Wang Z, Stanford EA, et al. An aryl hydrocarbon receptormediated amplification loop that enforces cell migration in $\mathrm{ER}^{-} / \mathrm{PR}^{-} /$ Her2 ${ }^{-}$human breast cancer cells. Mol Pharmacol 2016;90:674-88. https://doi.org/10.1124/mol.116.105361.

[35] Spranger S, Spaapen RM, Zha Y, et al. Up-regulation of PD-L1, IDO, and $\mathrm{T}(\mathrm{regs})$ in the melanoma tumor microenvironment is driven by $\mathrm{CD} 8^{+}$ T cells. Sci Transl Med 2013;5:200ra116. https://doi.org/10.1126/scitranslmed.3006504. 